\section{AB0580 CLINICAL CHARACTERISTICS AND RISK FACTORS OF BEHCET'S PATIENTS WITH CONDUCTION DISTURBANCES-DATA FROM A CARDIOVASCULAR CLINICAL CENTER IN CHINA}

R. Bai, N. Gao. Anzhen Hospital, Capital Medical University, Beijing, China

Background: Behcet's disease is a kind of systemic vasculitis affecting multiple systems. Although symptomatic cardiac involvement in Behcet's disease is uncommon, the fatality rate may reach up to $29 \%$. Conduction disturbances might lead to poor outcome and often need permanent pacemaker implanted. The characteristics of conduction disturbances in Behcet's disease were rarely reported before.

Objectives: To investigate the clinical characteristics of Behcet's patients with conduction disturbances and explore the underlying risk factors.

Methods: We reviewed 57 medical records of Behcet's disease with cardiovascular lesions in Anzhen Hospital, Capital Medical University between January 2002 and July 2015, and analyzed the clinical characteristics.

Results: (1) There were 13 patients with conduction disturbances out of a total of 57 Behcet's disease cases $(22.8 \%)$ in our research: three patients with first-degree atrioventricular block; one patient with Mobtizs type I second- degree atrioventricular block; four patients with third-degree atrioventricular block; one patient with the left anterior branch block; one patient with complete right bundle branch block; three patients with first-degree atrioventricular block combined with the left anterior branch block or complete right bundle branch block. One patient $(7.7 \%)$ died from infectious endocarditis and aortic root abscess during hospital stay.

(2) Hemoglobin level was significantly lower in cases than controls $(111.23 \pm 23.06$ vs. $127.16 \pm 19.86 \mathrm{~g} / \mathrm{L}, \mathrm{P}=0.017)$; The median of albumin level in cases was higher than controls [34.5 (14.2) vs. 38.5 (9.9) g/L, $P=0.032$ ] (Table 1); $C$ reactive protein level was higher in cases than controls [26.12 (21.3) vs. 6.7 (16.4) $\mathrm{mg} / \mathrm{L}, \mathrm{P}=0.045$ ] (Table 1)

(3) The aortic valvular insufficiency presented more frequently in patients in the case group than the control group ( $92.3 \%$ vs. $47.7 \%$, respectively; $P=0.004$ ). Heart reconstruction can be seen in Behcet'patients with cardiac involvement. The echocardiography showed the following parameters were significantly different between two groups: left ventricular end-diastolic diameter $(64.85 \pm 10.96$ vs. $52.55 \pm 10.13 \mathrm{~mm}, P=0.001$ ); left ventricular end-contraction diameter [48 (15) vs. 31 (13) mm, $P=0.001$ ]; left atrial diameter [50 (17) vs. 35.85 (13) mm, $P=0.003$; outflow tract of right ventricle [26 (7) vs. 27 (6) $\mathrm{mm}, \mathrm{P}=0.045]$

(4) Aortic valvular insufficiency was an independent risk factor for Behcet's disease with conduction disturbances $(\mathrm{OR}=1.157,95 \% \mathrm{Cl} 1.034,1.293, \mathrm{P}=0.011$ ).

Conclusions: Behcet's disease is one of the unusual etiologies of atrioventricular block. The evaluation of conduction disturbance should be kept in mind when diagnosing BD's patient.

References:

[1] Desbois A C, Wechsler B, Cluzel P, et al.: Cardiovascular involvement in Behcet's disease.Rev Med Interne 2014; 35(2):103-111.

[2] Braunwald E,Fauci A.S, Kasper D, et al.: Harrison's principles of international medicine. New York: McGraw-Hill 2001,1287.

[3] Butt SU, McNeil J.: Complete heart block in a Caucasian woman with Behçet's disease: a case report. J Med Case Rep 2016;19;10:102.

[4] Gao N, Han W, Ci WP, et al.: Clinical data analysis of cardiovascular involvement in Behcet's disease. Zhonghua Yi Xue Za Zhi 2016,24;96(19):1523-6.

Disclosure of Interest: None declared

DOI: 10.1136/annrheumdis-2017-eular.1240

\section{AB0581 TWO YEARS EXISTENCE OF REUMA.PT/VASCULITIS - THE PORTUGUESE REGISTRY OF VASCULITIS}

N. Khmelinskii ${ }^{1}$, C. Ponte ${ }^{1}$, D. Peixoto ${ }^{2}$, M. Rodrigues ${ }^{3}$, L. Teixeira ${ }^{4}$, S. Sousa ${ }^{4}$, J. Aleixo ${ }^{5}$, T. Pedrosa ${ }^{6}$, S. Serra ${ }^{6}$, W. Castelão ${ }^{6}$, A. Cordeiro ${ }^{4}$, I. Cordeiro ${ }^{4}$, S. Fernandes ${ }^{1}$, C. Macieira ${ }^{1}$, P. Madureira ${ }^{5}$, A. Malcata $^{3}$, V. Teixeira ${ }^{1}$, R. Vieira ${ }^{5}$, M. Eusébio ${ }^{7}$, F. Martins ${ }^{7}$, G. Sequeira ${ }^{8}$, J. Branco ${ }^{6}$, L. Costa ${ }^{5}$, J. Canas da Silva ${ }^{4}$, J.A. Pereira da Silva ${ }^{3}$, C. Afonso ${ }^{2}$, J.E. Fonseca ${ }^{1}$ H. Canhão ${ }^{7}$, R.A. Luqmani ${ }^{9}$, M.J. Santos ${ }^{4,7} .{ }^{1}$ Hospital Santa Maria-CHLN, Lisboa; ${ }^{2}$ Unidade Local de Saúde Alto Minho, Ponte de Lima; ${ }^{3}$ Centro Hospitalar Universitário de Coimbra, Coimbra; ${ }^{4}$ Hospital Garcia de Orta, Almada; ${ }^{5}$ Centro Hospitalar São João, Porto: ${ }^{6} \mathrm{Hospital}$ Egas Moniz-CHLO; ${ }^{7}$ Sociedade Portuguesa de Reumatologia, Lisboa; ${ }^{8}$ Hospital de Faro-CHA, Faro, Portugal; ${ }^{9}$ NDORMS, University of Oxford, Oxford, United Kingdom

Background: The vasculitides are a group of relatively rare diseases with different manifestations and outcomes. New therapeutic options have led to the need for long-term registries. The Rheumatic Diseases Portuguese Register, Reuma.pt, is an electronic clinical record, created in 2008, which currently includes specific protocols for 11 diseases and $>16000$ patients registered from 79 national and international rheumatology centres. Since October 2014, a dedicated protocol to vasculitis has been created as part of the European Vasculitis Society initiative of having compatible European registries.

Objectives: To describe the structure of Reuma.pt/Vasculitis and characterize the patients registered over the last two years.

Methods: We developed a dedicated web-based software to enable prospective collection and central storage of anonymised data from patients with vasculitis.
All Portuguese rheumatology centres were invited to participate. Data regarding demographics, diagnosis, classification criteria, imaging and laboratory tests, outcome measures of prognosis, damage, disease activity and quality of life, and treatment were collected. We performed a cross-sectional descriptive analysis of all patients registered up to January 2017.

Results: A total of 492 patients, with 1114 visits, from 11 centres were registered in Reuma.pt/Vasculitis. The mean age was $53 \pm 20$ years at last visit; $68 \%$ were females. The diagnoses followed the 2012 Chapel Hill Consensus nomenclature (Table 1). The most common diagnoses were Behcet's disease (BD) (39\%) and giant cell arteritis (GCA) (20\%). Patients with BD met the International Study Group 1990 criteria, the International Criteria for BD 2006 and 2013 in 84\%, $95 \%$ and $95 \%$ of cases, respectively. Patients with GCA met the 1990 American College of Rheumatology criteria in $95 \%$ of cases. Data on vascular ultrasound was available in $74 \%$ of patients; $73 \%$ compatible with the diagnosis. Assessment of the Birmingham Vasculitis Activity Score (BVAS) and Vasculitis Damage Index (VDI) was available for all vasculitides and the Five Factor Score calculation of survival rate for ANCA associated vasculitis (AAV) and polyarteritis nodosa (PAN). The mean BVAS at first visit was $18 \pm 7$ for AAV and 15 \pm 9 for PAN; the mean VDI at last visit was $3 \pm 2$ for $A A V$ and $2 \pm 2$ for PAN. Health related quality of life assessments (SF-36, EQD5, FACIT and HADS) were also collected. Treatment registry with the disease assessment variables shown in graphics was available for all patients; only $6 \%$ were under biologic treatment.
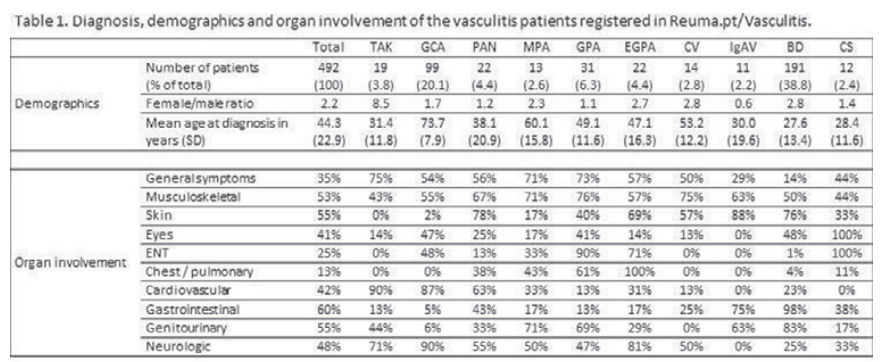

The patiens with single-organ vasulitis, vasculitis associated with spstemic disease, vasculitis associated with probable etiology, not-dassifiat

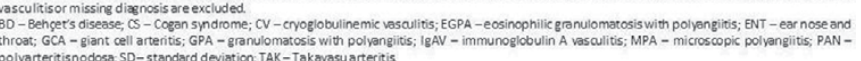

Conclusions: Reuma.pt/Vasculitis is a registry adapted for routine care, allowing an efficient data repository at a national level with the potential to link with other international databases. It facilitates research, trials recruitment, service planning and benchmarking.

Disclosure of Interest: None declared

DOI: 10.1136/annrheumdis-2017-eular.1815

\section{AB0582 ATHEROSCLEROTIC RISK FACTORS AND UPPER RESPIRATORY INFLAMMATIONS OF MPO-ANCA POSITIVE ANCA ASSOCIATED VASCULITIS}

N. Ono ${ }^{1}$, T. Miyamura ${ }^{2}$, Y. Inoue ${ }^{3}, \mathrm{~N}$. Ueki $^{4}, \mathrm{~K}$ Miyake $^{4}, \mathrm{~S} . \mathrm{Nagano}^{5}$ H. Inoue ${ }^{6}$, N. Ueda ${ }^{7}$, K. Oryoji $^{8}$, T. Sawabe $^{9}$, S. Yoshizawa ${ }^{10}$, Y. Takemoto ${ }^{11}$ Y. Kimoto ${ }^{12}$, S. Ohta $^{13}$, H. Nishizaka ${ }^{14}$, I. Furugo ${ }^{15}$, S. Yoshizawa ${ }^{2}$, H. Niiro ${ }^{16}$, T. Horiuchi ${ }^{12}$, H. Nakashima ${ }^{4}$, Y. Tada ${ }^{1} .{ }^{1}$ Rheumatology, Saga University, Saga; ${ }^{2}$ Rheumatology, National Fukuoka Hospital; ${ }^{3}$ Rheumatology, Fukuoka Red Cross Hospital; ${ }^{4}$ Rheumatology, Fukuoka University, Fukuoka; ${ }^{5}$ Rheumatology, lizuka Hospital, lizuka; ${ }^{6}$ Rheumatology, Saiseikai Fukuoka Hospital, Fukuoka; ${ }^{7}$ Rheumatology, Miyazaki Prefectural Miyazaki Hosipital, Miyazaki; ${ }^{8}$ Rheumatology, Matsuyama Red Cross Hospital, Matsuyama; ${ }^{9}$ Rheumatology, Hiroshima Red Cross Hospital, Hiroshima; ${ }^{10}$ Rheumatology, Hamanomachi Hospital, Fukuoka; ${ }^{11}$ Rheumatology, Saiseikai Karatsu Hospital, Karatsu; ${ }^{12}$ Rheumatology, Kyushu University Beppu Hospital, Beppu; ${ }^{13}$ Rheumatology, Shimonoseki City Hospital, Shimonoseki; ${ }^{14}$ Rheumatology, Kitakyushu Municipal Medical Center; ${ }^{15}$ Rheumatology, JR Kyushu Hospital, Kitakyushu;

${ }^{16}$ Rheumatology, Kyushu University, Fukuoka, Japan

Background: Recent studies had proven that the genetic backgrounds of ANCA associated vasculitis (AAV) were dependent on ANCAs. We and other groups had shown the differences between MPO-ANCA positive Granulomatosis with polyangitis (MPO-GPA) and Microscopic polyangitis (MPA) (1-3). It is not clear what determine these two phenotypes.

Objectives: To elucidate the etiologies of two phenotypes, we compared the backgrounds and comorbidities between MPO-GPA and MPA.

Methods: Retrospectively we recruited MPO-GPA and MPA patients through the two multi-center cohorts (Cohort A: 2001-2012, Cohort B: 2012-2016). We classified patients with EMEA classification and ANCA. We found 40 MPOGPA and 126 MPA cases without overlaps. We compared those backgrounds, comorbidities, organ involvements and outcomes.

Results: The average age of MPO-GPA group was similar to that of MPA (69.1 years old vs 72.1 years old). But MPO-GPA preferentially affected female patients $(80.0 \%$ vs $52.8 \%)$ with lower creatinine levels $(1.03 \mathrm{mg} / \mathrm{dl}$ vs $2.7 \mathrm{mg} / \mathrm{dl})$. Two year survivals of MPO-GPA were significantly better than MPA $(95.8 \%$ vs $73.2 \%, p=0.0424)$. Interestingly MPO-GPA patients had less atherosclerotic risk 